\title{
A Perturbation Finite Element Approach for Correcting Inaccuracies on Thin Shell Models with the Magnetic Field Formulation
}

\author{
Vuong Dang Quod*1, Quang Nguyen Duc ${ }^{2}$ \\ ${ }^{1}$ School of Electrical Engineering, Hanoi University of Science and Technology, Viet Nam \\ ${ }^{2}$ Faculty of Electrical Engineering, Electric Power University, Viet Nam, Viet Nam
}

A R T I C L E I N F O

Article history:

Received: 29 March, 2020

Accepted: 02 May, 2020

Online: 11 May, 2020

\section{Keywords:}

Magnetic field

Eddy current loss

Joule power loss

Thin shell model

Magnetodynamic

Subproblem technique

\section{A B S T R A C T}

This research makes the improvement of the errors surrounding edges and corners of thin electromagnetic regions by means of the magnetic field finite element formulation. Classical thin shell electromagnetic models are usually replaced by impedance-type interface conditions throughout surfaces that ignore errors in the calculation of the local fields (magnetic vector potentials, magnetic fields, eddy current densities and Joule power loss densities) in the vicinity of borders and corners. In this context, the inaccuracies of the local fields surrounding edges and curvatures related to the thin shell models are improved by a perturbation finite element technique, permitting to solve each subproblem on its own separately mesh and geometry, which makes reducing the computational time.

\section{Introduction}

Thin shell (TS) electromagnetic models have been presented by many author [1]-[3] to neglect meshing volumic shells (Fig. 1, left) and are introduced by surfaces (Fig. 1, right) with interface conditions (ICs) related to 1-D analytical distributions across the shell thickness.
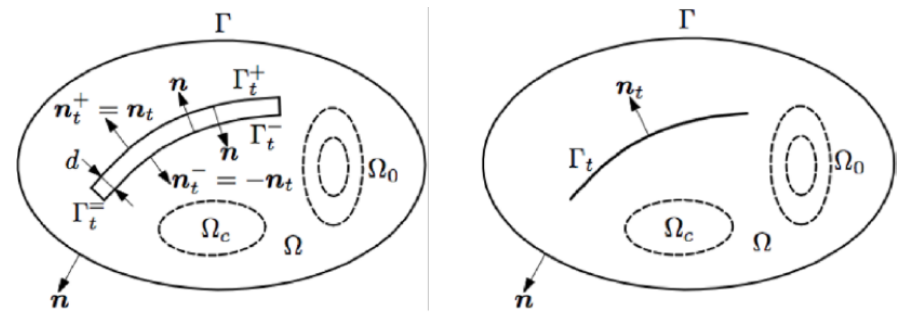

Figure 1: Volumic shell $\Omega_{t}($ left $)$ and IC $\Gamma_{t}$ (right).

In general, the ICs cancel corner and curvature effects, and lead to inaccuracies of magnetic fields, eddy current losses and Joule power losses in the neighbouring regions of geometrical discontinuities around borders and corners, changing with the thickness. So as to overcome with this disadvantage, a subproblem method (SPM) with a magnetic vector potential formulation for improving errors next to corners and curvatures occurring from the TS has recently presented by many author [4, 5].

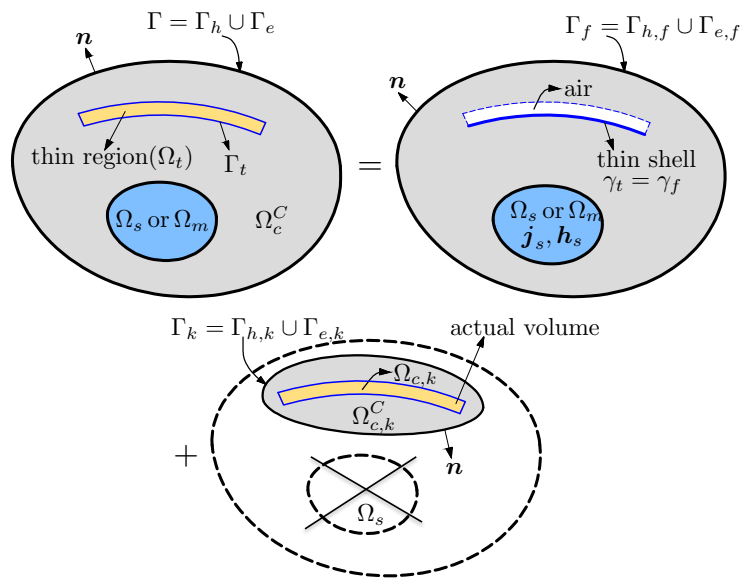

Figure 2: Decomposition of full problem into several SPs.

The idea of this article is to present an extension of the oneprocess SPM that proposes the magnetic field formulation. The hearth of this technique is based on a SPM, which consists in splitting a complete/full problem (including of stranded inductors and

*Corresponding Vuong Dang Quoc; Email: vuong.dangquoc@hust.edu.vn 
magnetic or conductive thin structures) into two subproblems (SPs), the complete/full solution being the superposition of all solutions for each SPs.

A problem involving stranded inductors and TS models (Fig. 2 . top right) is first considered on a simplified mesh. The obtained solution is then adjusted by a improvement problem with the actual volume thin regions (Fig. 2, bottom). In this process, each SP is constrained by volume sources (VSs) or surface sources (SSs), where VSs present variations of the permeability and conductivity in volume thin regions and SSs express variations of ICs across surfaces from previous SPs. Each process allows to inherit the previous sources/solutions for new SPs instead of starting a new complete problem for each new geometries as a traditional finite element method (FEM) [6].

\section{Sequence of Pertubation Technique}

\subsection{Magnetodynamic subproblem}

In the heart SPM, a canonical magnetodynamic SP $p$ considered at step $p$ is solved in a domain $\Omega_{p}$, with boundary $\partial \Omega_{p}=\Gamma_{p}=$ $\Gamma_{h, p} \cup \Gamma_{e, p}$. The eddy current density is defined in conducting part $\Omega_{c, p}$ of of $\Omega_{p}$, with $\Omega_{p}=\Omega_{c, p} \cup \Omega_{c, p}^{C}$ (where $\Omega_{c, p}^{C}$ is the nonconducting region. The set of maxwell's equations, constitutive laws and boundary conditions (BCs) of the SPs $p$ give [7, 8]:

$$
\begin{gathered}
\operatorname{curl} \boldsymbol{h}_{p}=\boldsymbol{j}_{p}, \operatorname{div} \boldsymbol{b}_{p}=0, \operatorname{curl} \boldsymbol{e}_{p}=-\partial_{t} \boldsymbol{b}_{p}, \\
\boldsymbol{b}_{p}=\mu_{p} \boldsymbol{h}_{p}+\boldsymbol{b}_{s, p}, \boldsymbol{e}_{p}=\sigma_{p}^{-1} \boldsymbol{j}_{p}+\boldsymbol{e}_{s, p}, \\
\boldsymbol{n} \times\left.\boldsymbol{h}_{p}\right|_{\Gamma_{h, p}}=\boldsymbol{j}_{s u, p}, \boldsymbol{n} \times\left.\boldsymbol{e}_{p}\right|_{\Gamma_{e, p} \subset \Gamma_{b, p}}=\boldsymbol{k}_{s u, p},
\end{gathered}
$$

$(1 \mathrm{a}-\mathrm{b}-\mathrm{c})$

where $\boldsymbol{h}_{p}$ is the magnetic field $(\mathrm{A} / \mathrm{m}), \boldsymbol{b}_{p}$ is the magnetic flux density (T), $\boldsymbol{e}_{p}$ is the electric field $(\mathrm{V} / \mathrm{m}), \boldsymbol{j}_{s, p}$ is the electric current density $\left(A / m^{2}\right), \mu_{p}$ is the magnetic permeability $(\mathrm{H} / \mathrm{m}), \sigma_{p}$ is the electric conductivity $(\mathrm{S} / \mathrm{m})$ and $\boldsymbol{n}$ is the unit normal exterior to $\Omega_{p}$. The source fields $\left(\boldsymbol{b}_{s, p}\right.$ and $\left.\boldsymbol{e}_{s, p}\right)$ in (2a-b) are VSs, and the source fields $\left(\boldsymbol{j}_{s u, p}\right.$ and $\left.\boldsymbol{k}_{s u, p}\right)$ in $3 \mathrm{a}-\mathrm{b}$ ) are SSs. In general, SSs are defined as zero for classical homogeneous BCs. ICs can express their discontinuities across the negative and positive sides $\left(\gamma_{p}^{+}\right.$and $\left.\gamma_{p}^{-}\right)$of any interface $\gamma_{p}$ (with the notation $[\cdot]_{\gamma_{p}}=\left.\right|_{\gamma_{p}^{+}}-\left.\right|_{\gamma_{p}^{-}}$) in $\Omega_{p}$. For the idealized thin regions, these ICs are SSs that have to account for special phenomena ocurring between two sides of $\gamma_{p}$ [4, 5].

\subsection{Relations of SPs with SSs and VSs}

As proposed in [3], the relation between SPs $p$ (TS models and volume corrections) are respectively defined via SSs and VSs. A volume thin region $\Omega_{t, p}$ in $\Omega_{c, p}$ is initially removed from $\Omega_{i}$ and then defined with the double layer TS surface $\Gamma_{t, p}$. For the magnetic field formulation ( $\boldsymbol{h}_{p}$-conformal formulation), the coefficient discontinuity $\boldsymbol{h}_{d, t, p}$ of the tangential component $\boldsymbol{h}_{t, p}=\left(\boldsymbol{n} \times \boldsymbol{h}_{p}\right) \times \boldsymbol{n}$ of $\boldsymbol{h}_{p}$ is presented across via the TS model, i.e. [3]

$$
\left[\boldsymbol{n} \times \boldsymbol{h}_{t, p}\right]_{\Gamma_{t, p}}=\boldsymbol{n} \times \boldsymbol{h}_{d, t, p},
$$

where the discontinuous component $\left(\boldsymbol{h}_{d, t, p}\right)$ of the field $\boldsymbol{h}_{t, p}$ is considered as zero along the TS border, which neglects the present magnetic fields. To present this discontinuity, it gets [3]

$$
\left.\boldsymbol{h}_{t, p}\right|_{\Gamma_{t, p}^{+}}=\boldsymbol{h}_{c, t, p}+\boldsymbol{h}_{d, t, p},\left.\boldsymbol{h}_{t, p}\right|_{\Gamma_{t, p}^{-p}}=\boldsymbol{h}_{c, t, p}
$$

where $\boldsymbol{h}_{c, p}$ is the continuous component of $\boldsymbol{h}_{p}$. The expressions (5) also applies on $\Gamma_{t, p}$ for the tangential components $\boldsymbol{h}_{t, p}, \boldsymbol{h}_{c, t, p}$ and $\boldsymbol{h}_{d, t, p}$.

The TS model combined with the ICs and BCs of the impedance BC type [3] are presented through $\boldsymbol{h}_{c, t, p}$ and $\boldsymbol{h}_{d, t, p}$, that is

$$
\begin{gathered}
{\left[\boldsymbol{n} \times \boldsymbol{e}_{p}\right]_{\Gamma_{t, p}}=\mu_{p} \beta_{p} \partial_{t}\left(2 \boldsymbol{h}_{c, t, p}+\boldsymbol{h}_{d, t, p}\right)} \\
\boldsymbol{n} \times\left.\boldsymbol{e}_{p}\right|_{\Gamma_{t, p}^{+}}=\frac{1}{2}\left[\mu_{p} \beta_{p} \partial_{t}\left(2 \boldsymbol{h}_{c, t, p}+\boldsymbol{h}_{d, t, p}\right)+\frac{1}{\sigma \beta} \boldsymbol{h}_{d, t, p}\right]-\boldsymbol{n} \times\left.\boldsymbol{e}_{p}\right|_{\Gamma_{t, p}^{-}} \\
\beta=\gamma_{p}^{-1} \tanh \left(\frac{d_{p} \gamma_{p}}{2}\right), \gamma_{p}=\frac{1+j}{\delta_{p}}, \delta_{p}=\sqrt{\frac{2}{\omega \sigma_{p} \mu_{p}}}
\end{gathered}
$$

where $d_{p}$ is the local thickness of the TS, $\delta_{p}$ is the skin depth, $\omega=$ $2 \pi f$ with $f$ is the frequency, $j$ is the imaginary unit and $\partial_{t} \equiv j \omega$. It should be noted that the term $-\boldsymbol{n} \times\left.\boldsymbol{e}_{i}\right|_{\Gamma_{t, i}^{-}}$in (7) is presented as a SS for the SPs.

As presented, the inaccuracy on the TS solution of a problem $p$ (e.g, $p=u$ ) is then improved by a volume correction SP $k$ (e.g., $p=$ $k$ ) which scopes with the TS hypothesis [3]. The changes from a TS region $\left(\mu_{u}\right.$ and $\sigma_{u}$ for SP $u$ ) to a volume thin region $\left(\mu_{k}\right.$ and $\sigma_{k}$ for SP $k$ ), the field sources $\left(\boldsymbol{h}_{s, k}\right.$ and $\left.\boldsymbol{j}_{s, k}\right)$ in $2 \mathrm{a}-\mathrm{b}$ ) are defined for the total fields [5, 8]

$$
\boldsymbol{b}_{s, k}=\left(\mu_{k}-\mu_{u}\right) \boldsymbol{h}_{u}, \quad \boldsymbol{e}_{s, k}=\left(\sigma_{k}^{-1}-\sigma_{u}^{-1}\right) \boldsymbol{j}_{u} .
$$

The equations in $9 \mathrm{a}-\mathrm{b}$ are then presented by the updated relations $\boldsymbol{b}_{u}+\boldsymbol{b}_{k}=\mu_{p}\left(\boldsymbol{h}_{u}+\boldsymbol{h}_{k}\right)$ and $\boldsymbol{e}_{u}+\boldsymbol{e}_{k}=\sigma_{p}^{-1}\left(\boldsymbol{j}_{u}+\boldsymbol{j}_{k}\right)$. Thus, for any number of sources of SPs, one can be written as

$$
\begin{gathered}
\boldsymbol{b}_{s, k}=\left(\mu_{k}-\mu_{u}\right) \sum_{q \in P, q \neq p} \boldsymbol{h}_{q}, \\
\boldsymbol{e}_{s, k}=\left(\sigma_{k}^{-1}-\sigma_{u}^{-1}\right) \sum_{q \in P, q \neq p} \boldsymbol{j}_{q},
\end{gathered}
$$

where $q \in P$ (total SPs) except the current SP $u$ relates to the sources $\left(\boldsymbol{b}_{s, k}\right.$ and $\boldsymbol{e}_{s, k}$ ) via their last calculated corrections of $\boldsymbol{h}_{q}$ and $\boldsymbol{j}_{q}$.

\section{Finite element weak formulation}

\section{$3.1 \boldsymbol{h}_{p}$-magnetic field formulation with source and re- action fields}

The weak magnetic field formulation ( $\boldsymbol{h}_{p}$-conformal formulation) is obtained via the Faraday's law (1c) [5, 8, 9]. The magnetic field $\boldsymbol{h}_{p}$ is decomposed into two parts, $\boldsymbol{h}_{p}=\boldsymbol{h}_{s, p}+\boldsymbol{h}_{r, p}$, where $\boldsymbol{h}_{s, p}$ is the source magnetic field presented via curl $\boldsymbol{h}_{s, p}=\boldsymbol{j}_{s, p}$, and $\boldsymbol{h}_{r, p}$ is the reaction magnetic field. In $\Omega_{c, p}^{C}$, the field $\boldsymbol{h}_{r, p}$ is expressed through a scalar potential $\phi_{p}$, i.e. $\boldsymbol{h}_{r, p}=-\operatorname{grad} \phi_{p}$. The weak forms for SPs $p$ $(p=u, k \ldots)$ are [10]

$$
\begin{aligned}
& \partial_{t}\left(\mu_{p}\left(\boldsymbol{h}_{r, p}+\boldsymbol{h}_{s, p}\right), \boldsymbol{h}_{p}^{\prime}\right)_{\Omega_{p}}\left(\sigma_{p}^{-1} \operatorname{curl} \boldsymbol{h}_{p}, \operatorname{curl} \boldsymbol{h}_{p}^{\prime}\right)_{\Omega_{c, p}}+\left(\boldsymbol{e}_{s, p}, \operatorname{curl} \boldsymbol{h}_{p}^{\prime}\right)_{\Omega_{p}} \\
&+\partial_{t}\left(\boldsymbol{b}_{s, p}, \boldsymbol{h}_{p}^{\prime}\right)_{\Omega_{i}}+\left\langle\boldsymbol{n} \times \boldsymbol{e}_{p}, \boldsymbol{h}_{p}^{\prime}\right\rangle_{\Gamma_{e, p}}+\left\langle\left[\boldsymbol{n} \times \boldsymbol{e}_{p}\right]_{\Gamma_{p}}, \boldsymbol{h}_{p}^{\prime}\right\rangle_{\Gamma_{p}}=0 \\
& \forall \boldsymbol{h}_{p}^{\prime} \in H_{p}^{1}\left(\Omega_{p}\right)
\end{aligned}
$$


where $H_{p}^{1}\left(\Omega_{p}\right)$ including the basis functions for $\boldsymbol{h}_{p}$ as well as for the test function $\boldsymbol{h}_{i}^{\prime}$ is a curl-conform function space presented in $\Omega_{p}$. Notation of $(\cdot, \cdot)_{\Omega_{i}}$ is volume integral in $\Omega_{p}$ and $<\cdot, \cdot>_{\Gamma_{p}}$ is the surface integral on $\Gamma_{p}$ of the product of their vector field arguments The term on surface integral $\Gamma_{e, p}$ gives as a natural BC of type (3 b), usually zero. It should be noted that the source field $\boldsymbol{h}_{s, p}$ in (12) is performed through a projection technique [11] of a known distribution $\boldsymbol{j}_{s, u}$, i.e.

$$
\left(\operatorname{curl} \boldsymbol{h}_{s, p}, \operatorname{curl} \boldsymbol{h}_{s, p}^{\prime}\right)_{\Omega_{p}}=\left(\boldsymbol{j}_{s, p}, \operatorname{curl} \boldsymbol{h}_{s, p}^{\prime}\right)_{\Omega_{s, p}}, \forall \boldsymbol{h}_{s, p}^{\prime} \in H_{p}^{1}\left(\Omega_{p}\right) .
$$

\subsection{Weak formulation for the TS model}

The TS model [3] occurred in the general equation (12) is defined a free discontinuous $\boldsymbol{h}_{d, t, u}(p=u)$ along the TS and $\boldsymbol{h}_{d, u}$ in the exterior region related to $\Gamma_{u}^{+}$and a $\mathrm{BC} \boldsymbol{n} \times\left.\boldsymbol{e}_{u}\right|_{\Gamma_{u^{+}}}$. This discontinuity is used as a test function $\boldsymbol{h}_{u}^{\prime}\left(\boldsymbol{h}_{u}^{\prime}=\boldsymbol{h}_{c, u}^{\prime}+\boldsymbol{h}_{d, u}^{\prime}\right)$ in $(12)$, where term contributions in the volume integrals in $\Omega_{u}$ are defined on the positive side of the TS. For that, the term $\left\langle\left[\boldsymbol{n} \times \boldsymbol{e}_{u}\right]_{\Gamma_{u}}, \boldsymbol{h}_{u}^{\prime}\right\rangle_{\Gamma_{u}}$ is analysed as

$$
\begin{aligned}
\left\langle\left[\boldsymbol{n} \times \boldsymbol{e}_{u}\right]_{\Gamma_{u}}, \boldsymbol{h}_{u}^{\prime}\right\rangle_{\Gamma_{u}}= & \left\langle\left[\boldsymbol{n} \times \boldsymbol{e}_{u}\right]_{\Gamma_{u}}, \boldsymbol{h}_{c, u}^{\prime}\right\rangle_{\Gamma_{u}}+\left\langle\boldsymbol{n} \times\left.\boldsymbol{e}_{u}\right|_{\Gamma_{u^{+}}}, \boldsymbol{h}_{d, u}^{\prime}\right\rangle_{\Gamma_{u^{+}}} \\
& +\left\langle\boldsymbol{n} \times\left.\boldsymbol{e}_{u}\right|_{\Gamma_{u^{-}}}, \boldsymbol{h}_{d, u}^{\prime}\right\rangle_{\Gamma_{u^{-}}},
\end{aligned}
$$

where with $\boldsymbol{h}_{d, u}^{\prime}$ is equal to zero on the negative side of TS $\Gamma_{u^{-}}$ [11]. Moreover, the term $\left\langle\left[\boldsymbol{n} \times \boldsymbol{e}_{u}\right]_{\Gamma_{u}}, \boldsymbol{h}_{c}^{\prime}\right\rangle_{\Gamma_{u}}$ and $\left\langle\boldsymbol{n} \times\left.\boldsymbol{e}_{u}\right|_{\Gamma_{u^{+}}}, \boldsymbol{h}_{d}^{\prime}\right\rangle_{\Gamma_{u^{+}}}$ are presented by (6) and (7), respectively. Therefore, (3.2) becomes

$$
\begin{gathered}
\left\langle\left[\boldsymbol{n} \times \boldsymbol{e}_{u}\right]_{\Gamma_{u}}, \boldsymbol{h}_{u}^{\prime}\right\rangle_{\Gamma_{u}}=\left\langle\mu_{u} \beta_{u} \partial_{t}\left(2 \boldsymbol{h}_{c, t, u}+\boldsymbol{h}_{d, t, u}\right), \boldsymbol{h}_{c, u}^{\prime}\right\rangle_{\Gamma_{u}}+ \\
\left\langle\frac{1}{2}\left[\mu_{u} \beta_{u} \partial_{t}\left(2 \boldsymbol{h}_{c, t, u}+\boldsymbol{h}_{d, t, u}\right)+\frac{1}{\sigma \beta} \boldsymbol{h}_{d, t, u}\right], \boldsymbol{h}_{d, u}^{\prime}\right\rangle_{\Gamma_{u^{+}}}
\end{gathered}
$$

By substituting the equation (15) into $(12)$, the weak formulation for the TS model is obtained

$$
\begin{gathered}
\partial_{t}\left(\mu_{u}\left(\boldsymbol{h}_{r, u}+\boldsymbol{h}_{s, u}\right), \boldsymbol{h}_{u}^{\prime}\right)_{\Omega_{u}}+\left(\sigma_{u}^{-1} \operatorname{curl} \boldsymbol{h}_{u}, \operatorname{curl} \boldsymbol{h}_{u}^{\prime}\right)_{\Omega_{c, u}} \\
+\left\langle\frac{1}{2}\left[\mu_{u} \beta_{u} \partial_{t}\left(2 \boldsymbol{h}_{c, t, u}+\boldsymbol{h}_{d, t, u}\right)+\frac{1}{\sigma \beta} \boldsymbol{h}_{d, t, i}\right], \boldsymbol{h}_{d, t, u}^{\prime}\right\rangle_{\Gamma_{u^{+}}} \\
+\left\langle\mu_{u} \beta_{u} \partial_{t}\left(2 \boldsymbol{h}_{c, t, u}+\boldsymbol{h}_{d, t, u}\right), \boldsymbol{h}_{c, t, u}^{\prime}\right\rangle_{\Gamma_{u}}+\left\langle\boldsymbol{n} \times \boldsymbol{e}_{u}, \boldsymbol{h}_{u}^{\prime}\right\rangle_{\Gamma_{e, u}-\Gamma_{t, u}}=0, \\
\forall \boldsymbol{h}_{i}^{\prime} \in H_{i}^{1}\left(\Omega_{i}\right)
\end{gathered}
$$

where the surface integral term on $\Gamma_{e, u}-\Gamma_{t, u}$ is usually considered as zero on a natural $\mathrm{BC}$.

\subsection{Projection of solutions between sub-models}

In the strategy SP technique, the solutions found from a previous problem SP $u$ are considered as sources (SSs and VSs) in a subdomain $\Omega_{s, k} \subset \Omega_{k}$ of the SP $k$ (current SP), e.g. from SP $u$ to SP $k$. At the discrete level, the field $\boldsymbol{h}_{u}$ in the mesh of the SP $u$ is transferred to the mesh ot the SP $k$ (i.e. $\boldsymbol{h}_{u, k-p r o j}$ ). This will be performed via a projection technique [11] of its curl limited to $\Omega_{s, k}$. The field $\boldsymbol{h}_{u, k-p r o j}$ is defined

$$
\left(\operatorname{curl} \boldsymbol{h}_{u, k-p r o j}, \operatorname{curl} \boldsymbol{h}^{\prime}\right)_{\Omega_{s, k}}=\left(\operatorname{curl} \boldsymbol{h}_{u}, \operatorname{curl} \boldsymbol{h}^{\prime}\right)_{\Omega_{s, k}}, \forall \boldsymbol{h}^{\prime} \in F_{k}^{1}\left(\Omega_{s, k}\right)
$$

where $H_{k}^{1}\left(\Omega_{s, k}\right)$ is a curl-conform function space containing the projection of $\boldsymbol{h}_{u}$ and the test function $\boldsymbol{h}^{\prime}$ in the mesh $k$. Directly projecting $\boldsymbol{h}_{u}$ instead of its curl will give numerical errors as estimating its curl.

\subsection{Improvement of the TS model}

The inaccuracies of the TS solution obtained in SP $u$ is now improved by the actual volume SP $k$ via VSs defined in (10) and (11). They are taken into account the changes from $\mu_{u}$ and $\sigma_{u}$ in the TS model to $\mu_{k}$ and $\sigma_{k}$ in the volume shell. These VSs also appear in (12) via the volume integrals $\left(\partial_{t}\left(\boldsymbol{b}_{s, k}, \boldsymbol{h}_{k}^{\prime}\right)_{\Omega_{k}}\right.$ and $\left.\left(\boldsymbol{e}_{s, k}, \operatorname{curl} \boldsymbol{h}_{k}^{\prime}\right)_{\Omega_{k}}\right)$. The weak form for SP $k$ is written as

$$
\begin{gathered}
\partial_{t}\left(\mu_{k} \boldsymbol{h}_{k}, \boldsymbol{h}_{k}^{\prime}\right)_{\Omega_{k}}+\left(\sigma_{k}^{-1} \operatorname{curl} \boldsymbol{h}_{k}, \operatorname{curl} \boldsymbol{h}_{k}^{\prime}\right)_{\Omega_{c, k}}++\left(-\boldsymbol{e}_{u}, \operatorname{curl} \boldsymbol{h}_{k}^{\prime}\right)_{\Omega_{k}}+ \\
\partial_{t}\left(\left(\mu_{k}-\mu_{u}\right) \boldsymbol{h}_{u}, \boldsymbol{h}_{k}^{\prime}\right)_{\Omega_{c, k}}+\left\langle\left[\boldsymbol{n} \times \boldsymbol{e}_{k}\right]_{\Gamma_{t, k}}, \boldsymbol{h}_{k}^{\prime}\right\rangle_{\Gamma_{k}}+\left\langle\boldsymbol{n} \times \boldsymbol{e}_{k}, \boldsymbol{h}_{k}^{\prime}\right\rangle_{\Gamma_{e, k}-\Gamma_{t, k}} \\
=0, \forall \boldsymbol{h}_{k}^{\prime} \in H_{k}^{1}\left(\Omega_{k}\right)
\end{gathered}
$$

where the field $\boldsymbol{e}_{u}$ is also performed via an electric problem [9]. As a sequence, the field $\boldsymbol{h}_{u}$ is transferred to the mesh of SP $k$ via (17), where $\Omega_{s, u}$ is limited to a single layer surrounding of the volumic shell. In the same time to the VSs in (12), SSs related to ICs [3], [5] have to extract the TS discontinuities, i.e, $\boldsymbol{h}_{d, k}=-\boldsymbol{h}_{d, u}$ and $\left[\boldsymbol{n} \times \boldsymbol{e}_{k}\right]_{\Gamma_{t s, k}}=-\left[\boldsymbol{n} \times \boldsymbol{e}_{u}\right]_{\Gamma_{t s, u}}$. The involved trace $\left[\boldsymbol{n} \times \boldsymbol{e}_{u}\right]_{\Gamma_{t s, k}}$ is generally defined through other integrals in (12), with $\Gamma_{t s, u}=\Gamma_{t s, k}$, i.e.

$$
\begin{aligned}
\left\langle\left[\boldsymbol{n} \times \boldsymbol{e}_{k}\right]_{\Gamma_{t s, k}}, \boldsymbol{h}_{k}^{\prime}\right\rangle_{\Gamma_{t s, k}}= & \left\langle-\left[\boldsymbol{n} \times \boldsymbol{e}_{u}\right]_{\Gamma_{t s, k}}, \boldsymbol{h}_{k}^{\prime}\right\rangle_{\Gamma_{t s, k}}=\left(\mu_{u} \partial_{t} \boldsymbol{h}_{s, u}, \boldsymbol{h}_{k}^{\prime}\right)_{\Omega_{u}=\Omega_{k}} \\
& +\left(\mu_{u} \partial_{t} \boldsymbol{h}_{u}, \boldsymbol{h}_{k}^{\prime}\right)_{\Omega_{u}=\Omega_{k}}
\end{aligned}
$$

The surface integral in (19) is implemented in the step $u$. At the discrete level, the limitation of the two volume integrals in (19) is defined in a single layer of FEs on both sides of $\Gamma_{t, u}$ touching $\gamma_{k}^{+}=\gamma_{u}^{+}=\Gamma_{t}^{+}$, because it involves only the trace $\boldsymbol{n} \times\left.\boldsymbol{h}_{k}^{\prime}\right|_{k} ^{+}$.

\section{Numerical test}

The practical test is a TEAM workshop problem (problem 21, model B) [12] including two coils and a magnetic shielding plate (Fig. 3]. The test problem is considered in both 2-D and 3-D models.

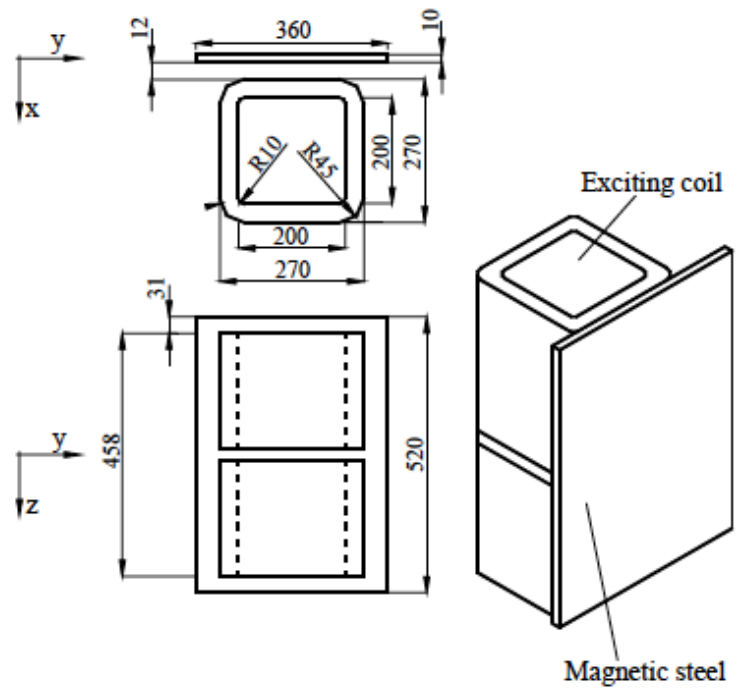

Figure 3: Geometry of the test problem 21 (model B). 


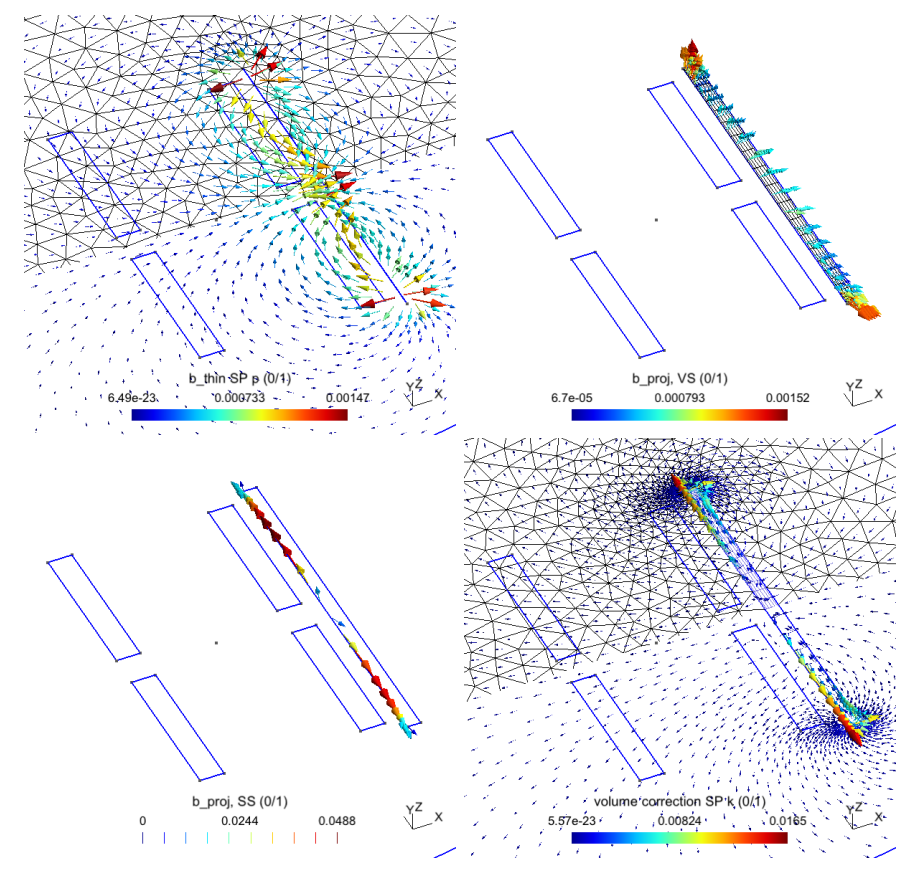

Figure 4: Magnetic flux densities for the TS solution SP $u\left(\boldsymbol{b}_{u}\right.$, top left $)$ and volume correction solution SP $k\left(\boldsymbol{b}_{k}\right.$, bottom right). Projections of SP $u$ solutions $\left(\boldsymbol{b}_{\text {proj }}\right.$, VS, top right) and ( $\boldsymbol{b}_{\text {proj }}, \mathrm{SS}$, bottom left $)$ in the SP $k$.
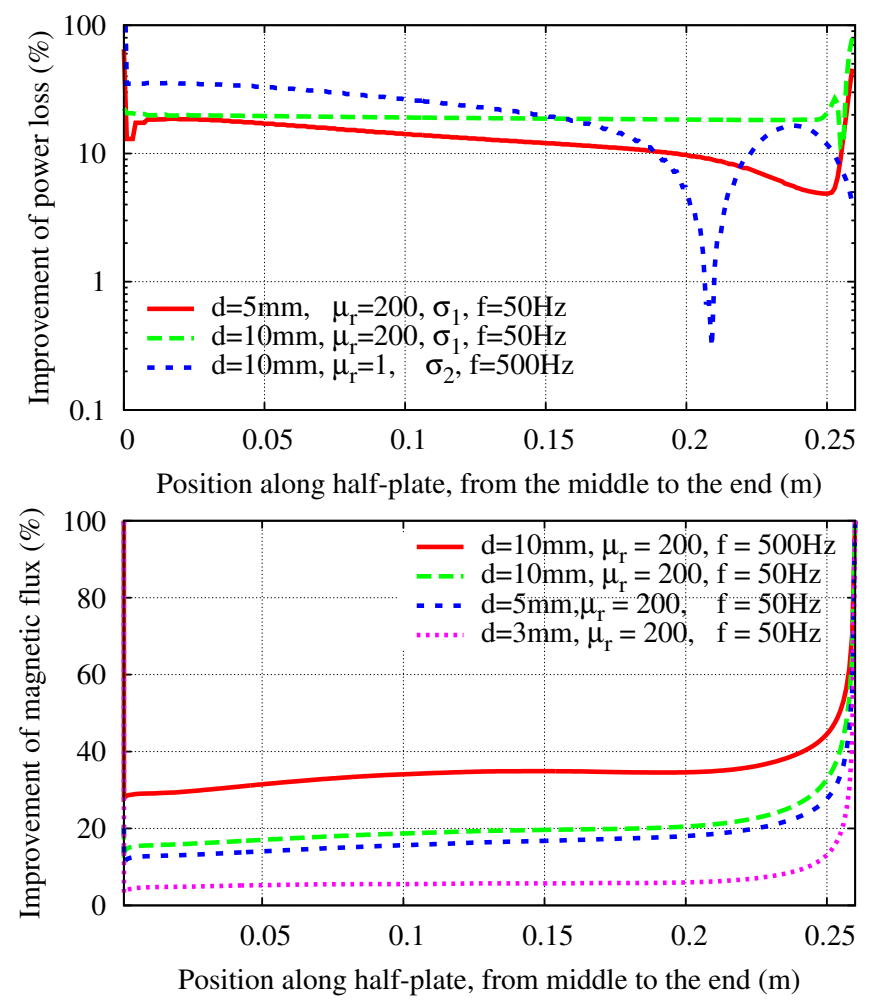

Figure 5: Relative improvements of the joule power loss density (top) and the magnetic flux (bottom) along the thin plate, with $\sigma_{\text {plate }}=6.484 \mathrm{MS} / \mathrm{m}$.

The first case is tested with a 2-D model: The SP $u$ with the inductors and TS FE magnetodynamic model is solved on a lighter mesh (Fig. 4( $\left.\boldsymbol{b}_{u}\right)$, top left). A volume correction SP $k$ then replaces the TS FEs with the classical volume FEs containing an actual re- gion of the thin plate and its surrounding, with a suitable refined mesh without including the inductors anymore (Fig. $4\left(\boldsymbol{b}_{k}\right)$, bottom right). The projections of TS solution in TS SP $u$ for the VS and SS are respectively pointed out (Fig. 4 ( $\boldsymbol{b}_{\text {proj }}$, VS), top right) and (Fig. 4 ( $\boldsymbol{b}_{\text {proj }}$, SS), bottom left). The relative improvements of the joule power loss density and the magnetic flux along the plate are depicted in Fig. 5. They can touch several tens of percents near the end of the TS, such as $80 \%$ (Fig. 5. top) and 85\% (Fig.5. bottom), with $d=10 \mathrm{~mm}$ and $\delta=1.977 \mathrm{~mm}$ for both cases. For $d=10$ $\mathrm{mm}$, the inaccuracy is lower than 50\% (Fig. 5. top) and 55\% (Fig. 5 . bottom).
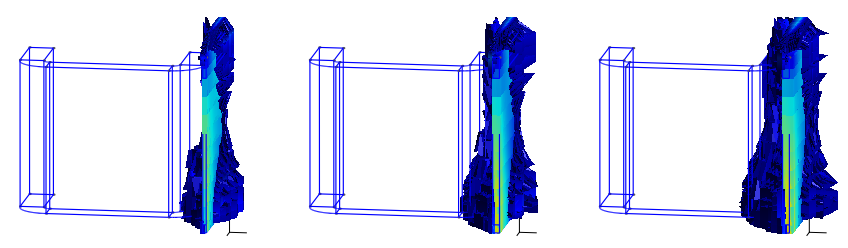

Figure 6: Colored maps of eddy current densities indicating the regions with relative volume improvements greater than $2 \%$ for $d=5 \mathrm{~mm}, 7.5 \mathrm{~mm}$ and $10 \mathrm{~mm}$ (from left to right $), f=50 \mathrm{~Hz}, \mu_{\text {plate }}=200$ and $\sigma_{\text {plate }}=6.484 \mathrm{MS} / \mathrm{m}$.
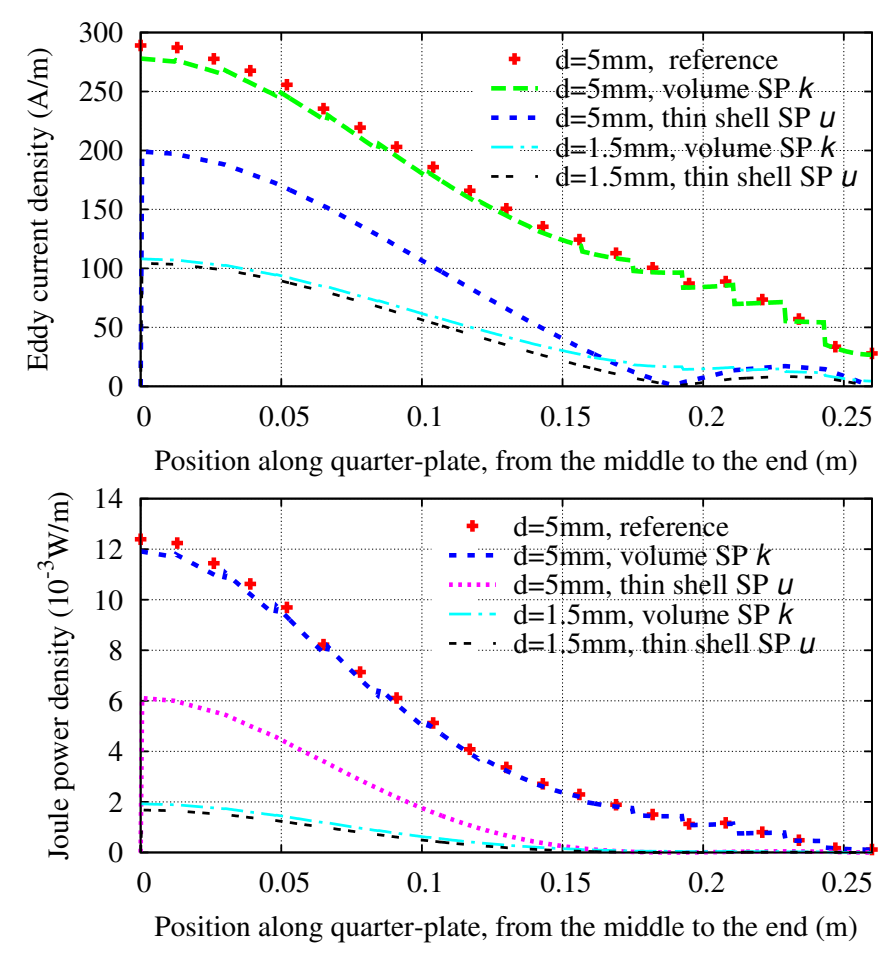

Figure 7: Eddy current density (top) and joule power loss density (bottom) for the TS and the volume corection along the vertical half edge (z-direction) $(f=50 \mathrm{~Hz}$, $\mu_{\text {plate }}=200$ and $\sigma_{\text {plate }}=6.484 \mathrm{MS} / \mathrm{m}$ ).

The next test is considered with a 3-D model: The errors of the TS model SP $u$ are shown in Fig. 6(from left to right). They increase with plate thickness, specially near the ends of the plate, are perfectly improved whatever their order of magnitude. The accuracy of the improvement is directly related to the volume correction of the plate and its surrounding. 
The inaccuracies on the eddy current density and joule power loss density of TS model (SP $u$ ) are improved by the significant volume correction (SP $k$ ) (Fig. 7. The inaccuracies on the local fields (eddy current density and joule power loss density) along the vertical half edge ( $z$-direction) near the plate ends reaches $60 \%$ (Fig.77. top) and $80 \%$ (Fig. 7. bottom), respectively, with $\delta=2.1 \mathrm{~mm}$ and $d=5 \mathrm{~mm}$. The volume corrections are then compared to be similar the reference solutions (obtained from the FEM) for different parameters in both cases.

\section{Conclusions}

In this study, a perturbation Finite Element Approach has been proposed with a magnetic field formulation for a one-way coupling in order to improve the errors around edges and corners inherent to the TS FE assumptions. The correctly improving on the local fields of magnetic flux densities, eddy current losses and joule power losses is successfully achieved surrounding the borders and corners of the TS structures. The proposed method has been developed for the one-way coupling in two steps. All the processes of the method have been successfully validated on the international practical problem (TEAM workshop problem 21, model B) [12].

The source-codes of the this technique have been being extended based on the source-codes of the SPM that was developing by author and two Prof. Patrick Dular and Prof. Christophe Geuzaine at the Dept of Electrical Engineering and Computer Science, University of Liege, Belgium. They will be then ran and simulated in the background of the Getdp (http://getdp.info) and Gmsh (http://gmsh.info) softwares developed by Prof. Patrick Dular and Prof. Christophe Geuzaine [13, 14]. These are the open-source codes for anyone to be able to write adapt source-codes for solving studied problems (if possible).

\section{References}

[1] L. Krähenbühl and D. Muller, "Thin layers in electrical engineering. Examples of shell models in analyzing eddy- currents by boundary and finite element methods," IEEE Trans. Magn., vol. 29, no. 2, pp. 1450-1455, 1993.
[2] Tsuboi, H., Asahara, T., Kobaysashi, F. and Misaki, T. (1997), "Eddy current analysis on thin conducting plate by an integral equation method using edge elements," IEEE Trans. Magn., Vol. 33, No. 2, pp. 1346-9.

[3] C. Geuzaine, P. Dular, and W. Legros, "Dual formulations for the modeling of thin electromagnetic shells using edge elements," IEEE Trans. Magn., vol. 36, no. 4, pp. 799-802, 2000.

[4] P. Dular, Vuong Q. Dang, R. V. Sabariego, L. Krähenbühl and C. Geuzaine, "Correction of Thin Shell Finite Element Magnetic Models via a Subproblem Method,” IEEE Trans. Magn., vol. 47, no. 5, pp. 1158-1161, 2011.

[5] Vuong Q. Dang, P. Dular, R. V. Sabariego, L. Krähenbühl and C. Geuzaine, "Subproblem Approach for Thin Shell Dual Finite Element Formulations," IEEE Trans. Magn., vol. 48, no. 2, pp. 407-410, 2012.

[6] S. Koruglu, P. Sergeant, R.V. Sabarieqo, Vuong. Q. Dang and M. De Wulf "Influence of contact resistance on shielding efficiency of shielding gutters for high-voltage cables," IET Electric Power Applications., Vol. 5, No. 9, pp.715-720, 2011.

[7] Vuong Quoc Dang and Quang Nguyen Duc, "Coupling of Local and Global Quantities by A Subproblem Finite Element Method Application to Thin Region Models," ASTESJ, vol. 4, no. 2, pp. 40-44, 2019.

[8] Vuong Quoc Dang and Quang Christophe Geuzaine, "Using edge elements for modeling of 3-D Magnetodynamic Problem via a Subproblem Method," Sci. Tech. Dev. J.; 23 (1), pp. 439-445, 2020.

[9] P. Dular and R. V. Sabariego, "A perturbation method for computing field distortions due to conductive regions with h-conform magnetodynamic finite element formulations," IEEE Trans. Magn., vol. 43, no. 4, pp. 1293-1296, 2007.

[10] Vuong Q. Dang, P. Dular, R. V. Sabariego, L. Krähenbühl, C. Geuzaine "Subproblem Approach for Modelding Multiply Connected Thin Regions with an h-Conformal Magnetodynamic Finite Element Formulation," in EPJ AP., vol. 63, no. 1, 2013.

[11] C. Geuzaine, B. Meys, F. Henrotte, P. Dular and W. Legros, "A Galerkin projection method for mixed finite elements," IEEE Trans. Magn., Vol. 35, No. 3, pp. 1438-1441, 1999.

[12] Zhiguang CHENG1, Norio TAKAHASHI2, and Behzad FORGHANI, "TEAM Problem 21 Family (V.2009)," Approved by the International Compumag Society Board at Compumag-2009, Florianopolis, Brazil.

[13] C. Geuzaine and J.-F. Remacle "Gmsh: a three-dimensional finite element mesh generator with built-in pre- and post-processing facilities," International Journal for Numerical Methods in Engineering 79(11), pp. 1309-1331, 2009.

[14] P. Dular and C. Geuzaine "GetDP reference manual: the documentation for GetDP, a general environment for the treatment of discrete problems," University of Liege, 2013. 